\title{
Determination of Transcoronary Girculatory Transport Function
}

\author{
Kai Tsuiki, Junichiro Nagasawa and Takashi Nakamura \\ The First Department of Internal Medicine, Yamagata \\ University School of Medicine, Yamagata
}

Tsuiki, K., Nagasawa, J. and Nakamura, T. Determination of Transcoronary Circulatory Transport Function. Tohoku J. exp. Med., 1977, 122 (1), 43-50 - A method is described to determine the transcoronary circulatory transport function $(h(t))$ from input $\left(C_{i}^{\prime}(t)\right)$ and output $\left(C_{\circ}^{\prime}(t)\right)$ dye-dilution curves obtained at the inlet and outlet of the coronary circulation. Assuming the mathematical linearity and stationarity of the coronary circulation, it is demonstrated that $h(t)$ can be computed in terms of lagged normal density curve as a model from the sole measurement of the first to third moments of $C_{i}{ }^{\prime}(t)$ and $C_{o}{ }^{\prime}(t)$ when recorded by a pair of our dye sampling systems that have shown to have identical response to step function. The method is useful because of its simplicity in practice. The physiological meaning of the determination of $h(t)$ is discussed. It could be helpful to the estimation of the change in coronary path-length distribution under some conditions, although the method is still of limited value at present, because the averaged path-length through the coronary circulation cannot be evaluated correctly. - dye-dilution method; mean transit time; coronary circulation; path-length distribution

The output response of a system to unit impulse is transfer function (Stephenson 1948; Meier and Zierler 1954). When a circulatory system is involved, it is called circulatory transport function (Bassingthwaighte 1970), that is the probability density function of transit times through the system. Since extensive studies of Bassingthwaighte and co-investigators (Bassingthwaighte 1966; Coulam et al. 1966; Bassingthwaighte et al. 1966; Bassingthwaighte and Ackerman 1967; Knopp and Bssingthwaighte 1969), the physiological meaning of transport function is being clarified; the ratio of the mean transit time to the standard deviation of transport function (called relative dispersion) is constant for a simply perfused system such as aorta and branching arteries even with the flow changing by 6 times (Bassingthwaighte 1966), but it is variable in a more complicated-structured system (Knopp and Bassingthwaighte 1969), so that in pulmonary circulatory bed, it varied with increased flow suggesting that an alteration in perfusion homogeneity has occurred. Knowledge of the path-length distribution seems very important for the clinical purpose in coronary circulation, in particular in the assessment of the development of collateral circulation, for under such conditions the path-length distribution may be altered (Liedtke et al. 1973).

Received for publication, October 23, 1976. 
Although the determination of transport function has been recognized to be laborious and difficult (Bassingthwaighte 1966, 1970; Gamel et al. 1973), its lower moments can be calculated relatively easily (Lilienfield and Kovach 1956). The present report describes a simple method to determine the transcoronary circulatory transport function from the sole measurement of the moments of input and output dye dilution curves obtained at the inlet and outlet of the coronary circulation. The method is applicable to any circulatory system, but this article deals with an application to coronary circulation. The second purpose of this paper is to discuss the physiological meaning of the moment-analysis in estimating path-length distribution of the coronary circulation.

\section{Methods}

\section{Determination of circulatory transport function}

Assuming that the coronary circulatory system is mathematically linear and the flow through it is steady, the transport function results in the solution of convolution integral of input and output dye-dilution curves. Because it is impossible to make an ideal unit impulse of dye concentration-versus-time curve in the circulation, it is reasonable to derive the impulse response (transport function, $h(t)$ ) using the following relation:

$$
C_{o}(t)=h(t) ※ C_{i}(t)
$$

in which $C_{i}(t)$ and $C_{o}(t)$ are the input and output dilution curves, respectively, which are obtained at the inlet and outlet of the system after a rapid injection of dye bolus into an upstream point of the circulation, and $\%$ indicates the process of convolution. It has been shown (Bassingthwaighte and Ackerman 1967) that when two identically responding sampling systems are used to record $C_{o}(t)$ and $C_{i}(t)$, the resulting curves, $C_{o}^{\prime}(t)$ and $C_{i}{ }^{\prime}(t)$, hold the following relation with $h(t)$ :

$$
C_{o}^{\prime}(t)=h(t) ※ C_{i}^{\prime}(t)
$$

Solving equation (2), $h(t)$ may be computed. The major problem is that one does not know the mathematical description of the function for $C_{o}{ }^{\prime}(t)$ and $C_{i}{ }^{\prime}(t)$, so that $h(t)$ is also unknown. Only by fitting the curves to some known mathematical form, $h(t)$ can be defined and determined. If a three-parameter model such as lagged normal density curve (Bassingthwaighte et al. 1966) is applied to $h(t)$, then $h(t)$ can be computed from calculating only the first to third moments of $C_{o}^{\prime}(t)$ and $C_{i}^{\prime}(t)$, without knowing the mathematical form for them provided that the recirculation of dye is eliminated. The first and second moments of the dye curve are meaningful, and called mean transit time $(\bar{l})$ and variance $\left(\pi_{2}\right)$, respectively. The third moment $\left(\mu_{3}\right)$ is also useful, while the higher moments are useless (Bassingthwaighte 1970). Since each moment is simply additive by definition (Cramér 1946), each moment for $h(t)$ can be calculated from that for $C_{0}^{\prime}(t)$ and $C_{i}{ }^{\prime}(t)$ under the conditions where equation (2) holds as follows:

$$
\begin{aligned}
& i_{h(l)}=i_{C_{0^{\prime}}(l)}-i_{C_{i^{\prime}(b)}}=\Delta i \\
& \pi_{z_{h(t)}}=\pi_{2 c_{o^{\prime}(t)}}-\pi_{2 c_{i^{\prime}(t)}}=\Delta \pi_{2} \\
& \mu_{3 h(l)}=\mu_{3 c_{o^{\prime}(1)}}-\mu_{3 c_{i^{\prime}(1)}}=\Delta \mu_{3}
\end{aligned}
$$

In the lagged normal density curve model, there are three unknown parameters, $t_{c}, \sigma$ and $\tau$, all of which can be calculated from the three moments listed above as follows:

$$
\begin{aligned}
& t_{c}=\Delta \bar{l}-\tau \\
& \sigma=\left(\Delta \pi_{2}-\tau^{2}\right)^{1 / 2} \\
& \tau=\left((1 / 2) \cdot \mu_{3}\right)^{1 / 3}
\end{aligned}
$$


Note that this approach to compute $h(t)$ requires only one assumption in the calculation that transport function can be written in terms of lagged normal density curve. Any other model may be used.

\section{Animal experiment}

Adult mongrel dog was anesthetized with pentobarbital sodium intravenous injection at the rate of $25 \mathrm{mg} / \mathrm{kg}$. The trachea was intubated and artificial breathing was performed. The dog was thoractomized at the fifth intercostal space bilaterally. The pericardium was incised, and the heart was suspended on the pericardium in a cradle position. The right atrium was incised, and a specially shaped polyethylene tube (about $15 \mathrm{~cm}$ long) was inserted and wedged in the coronary sinus, about $1 \mathrm{~cm}$ proximal to its right atrial orifice. We prepared several tubes with different diameters. Selection of the size was done in a trial-and-error manner. Blood from the tube was accumulated in a small reservoir, and returned to the femoral vein with a constant infusion pump. This extracorporeal circulation is required to measure coronary sinus outflow, when nesseccary. 2,000 units of heparin were administered intravenously. A $9 \mathrm{~F}$ Gensini catheter was introduced via femoral artery into the ascending aorta, and positioned just above the aortic valve, where the location of the tip was verified by digital palpation. Another $9 \mathrm{~F}$ Gensini type catheter was placed in the coronary sinus tube. These catheters were connected to a pair of cuvettes of dye-densitometers. A US catheter $(6 \mathrm{~F})$ was introduced from the other side of femoral artery into the ascending aorta for the recording of the aortic blood pressure. A $6 \mathrm{~F}$ tube was introduced in the femoral vein, which was used to add blood.

The tightness of the coronary sinus tube was tested by injection of dye-bolus from the femoral vein catheter and recording dye-concentration at the coronary sinus, or by recording from aortic catheter following coronary sinus injection of dye, whereby the sampling and injection were set at the usual rate as described below. When no dye appeared in the first circulation on each record, the coronary sinus cannulation was considered to be appropriate. The test was repeated during the experiment whenever a leakage was suspected.

The cuvettes of the dye-densitometers were connected to a dual infusion/withdrawal pump (Harvard), by which the blood was withdrawn at a constant rate of $19 \mathrm{ml} / \mathrm{min}$ from two calibrated $200 \mathrm{ml}$ syringes, simultaneously. Cardiogreen dye $(5 \mathrm{mg} / \mathrm{ml})$ was injected by hand as a $0.5 \mathrm{ml}$ bolus into the inferior vena cava, and the dye concentration-versus-time curves were recorded simultaneously from the coronary sinus and the aortic root on a direct pen recorder, along with ECG and aortic and left ventricular pressures.

Care was taken to hold the blood volume of the dog as constant as possible during the experiment.

\section{Calibration of dye-curve recording systems}

Calibration of the dye densitometers was performed by the whole blood technique with one-point check, because the linearity was already established within the range of dye amount used.

The catheter (9F Gensini) - cuvette system was tested for its dynamic characteristics as follows: A step change in the dye concentration was produced by a rapid manual turn of a 3 -way cock during the pump-withdrawing of the blood, and the resulting response by the two systems was compared.

\section{Analysis of dye curves}

Since mathematical linearity and stationarity may be assumed without producing significant error in the calculations for circulatory systems (Bassingthwaighte and Ackerman 1967), the moments of $h(t)$ can be calculated using equations (3)-(5), and the shape of the $h(t)$ may be computed using equations $(6)-(8)$ on the basis of lagged normal density curve as a model (Bassingthwaighte et al. 1966), provided that the equation (2) holds. 
Dye curves recorded were replotted on semi-logarithmic paper, and the downslope $\left(t_{s}\right)$ was calculated according to Hamilton's method (Kinsman et al. 1929). The area of the first circulating dye curves, $\bar{i}$, and $\pi_{2}$ were calculated by the Lilienfield and Kovach method (1956). The first to third moments of $h(t)$ were calculated using equations (3)-(5), and the three parameters of lagged normal density curve model were calculated using equations (6)-(8). The instantaneous values for $h(t)$ as based on the model were computed twice a second according to the method of Bassingthwaighte et al. (1966) utilizing a digital computer, and the shape of its curve was displayed on an oscilloscope.

\section{Results}

Dynamic response of dye curve recording systems used

Fig. 1 shows the dynamic response to a step change in the dye concentration recorded with the two recording systems. Since there was an agreement in the response in general, it was considered that the two systems are identical in dynamic response of measuring the dye concentration. Therefore, equation (2) holds with our densitometry systems.

\section{Computation of the transport function and the moments}

Fig. 2 shows a pair of input and output curves actually recorded and the transport function computed therefrom in terms of lagged normal density curve model.

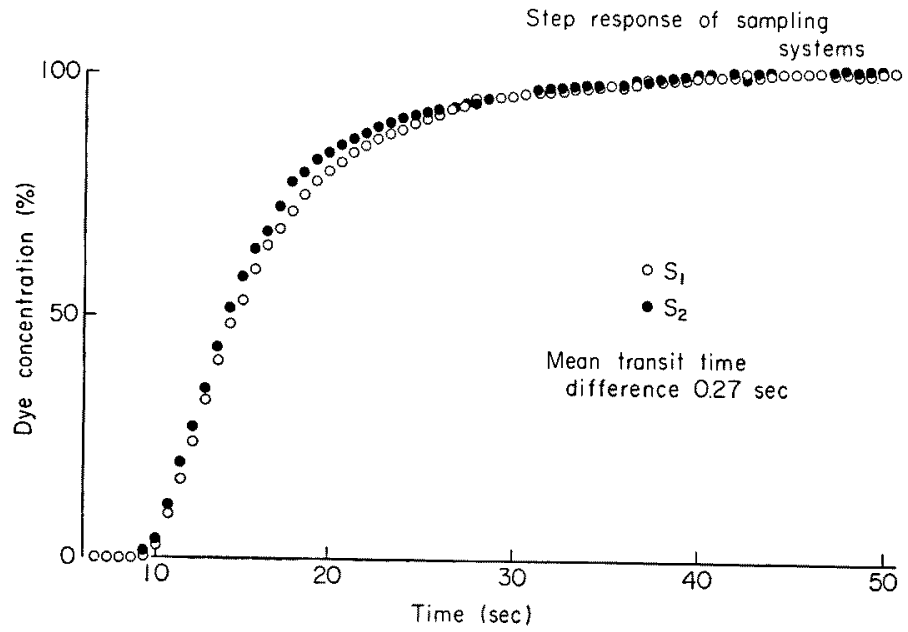

Fig. 1. Test for the dynamic response of the dye recording catheter-cuvette densitometerrecorder systems used in the present study. Note that two systems $\left(\mathrm{S}_{1}\right.$ and $\left.\mathrm{S}_{2}\right)$ show identical response to a step change in the dye concentration.

\section{Discussion}

Theoretical considerations of the physiological significance of moment-analysis of transport function.

Let us assume that Taylor's (1953) one dimensional three parameter model, that is, 


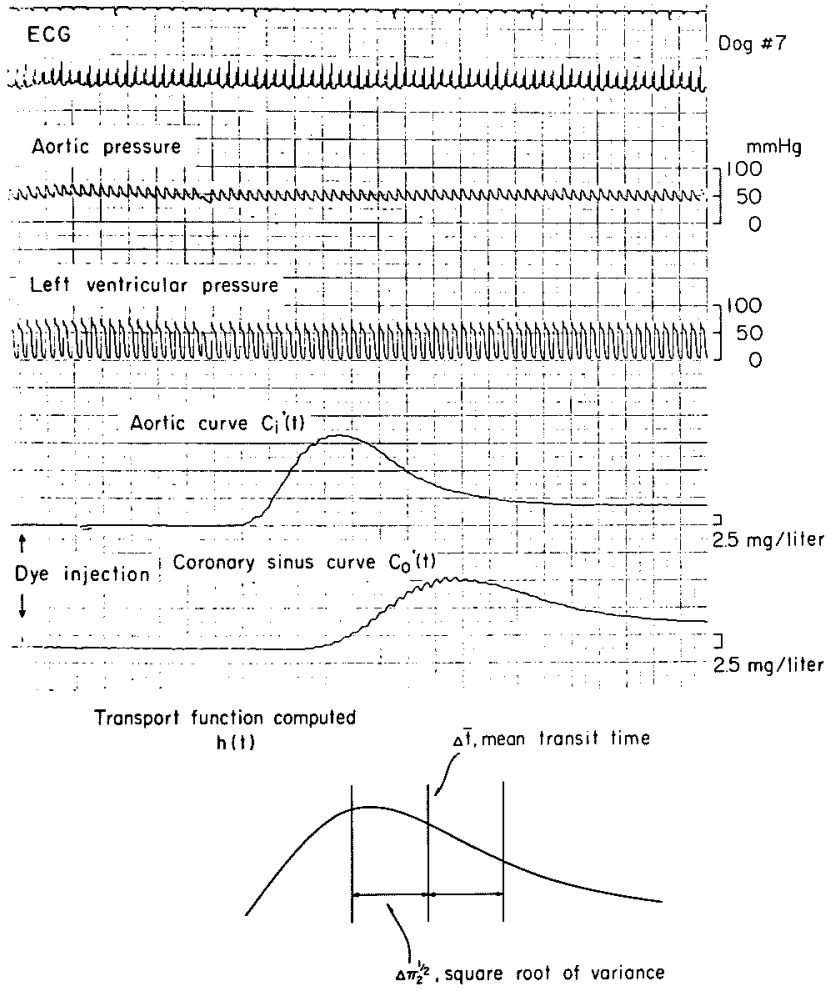

Fig. 2. Experimental recordings of dye-dilution curves, and simultaneously measured electrocardigoram (ECG), and aortic and left ventricular pressures (upper). Two dye curves, $C_{i}{ }^{\prime}(t)$ and $C_{0}{ }^{\prime}(t)$ were recorded with $S_{1}$ and $S_{2}$ of Fig. 1 , at the inlet and outlet of the coronary circulation, respectively. Transcoronary circulatory transport function $(h(t))$ computed from the $C_{i}{ }^{\prime}(t)$ and $C_{o}{ }^{\prime}(t)$ is shown in the lower. Lagged normal density curve is used as a model for $h(t)$.

$$
\frac{\partial c}{\partial t}=D \frac{\partial^{2} c}{\partial x^{2}}-v \frac{\partial c}{\partial x}
$$

in which $t$ is time, $c$ is dye concentration, $x$ is distance, $D$ is diffusion coefficient, and $v$ is velocity, is applicable to the coronary circulatory bed. The solution of the equation (9) is given by Norwich and Zelin (1970) as;

$$
C(t)=\frac{m}{\sqrt{4 \pi D A^{2}}} t^{-1 / 2} \exp \left[-\frac{\left(v A^{2}\right)}{4 D A^{2}}\left(\frac{t-x_{0} / v}{t}\right)\right]
$$

in which $m$ is mass of indicator, $x_{0}$ is a distance downstream from the dye injection site, and $A$ is mean cross sectional area of the vessels. If one takes the first and second moments from $x=0$ through $x=1$, then the moments of $h(t)$ can be written in terms of diffusion coefficient $(D)$, velocity $(v)$, and length $(l)$ as follows:

$$
\begin{aligned}
& \Delta \bar{t}=l / v \\
& \Delta \pi_{\mathbf{2}}=(2 \cdot l / D v)^{2}
\end{aligned}
$$


The same equations are obtained from the results of Wingard et al. (1972) who derived the relationships in a dimensionless form. Relation of equation (11) has been recognized, with multiplying $A$ to the denominator and nominator;

$$
\Delta \bar{t}=\frac{l A}{v A}=\frac{V}{F}
$$

in which $F$ is flow $(\mathrm{ml} / \mathrm{min})$ and $V$ is vascular volume $(\mathrm{ml})$.

From equations (11) and (12), the relative dispersion $\left(\Delta \pi_{2}^{1 / 2} / \Delta \bar{t}\right)$ can be evaluated in terms of $c, D$, and $l$.

$$
\frac{\Delta \pi_{2}^{1 / 2}}{\Delta \bar{t}}=\left(\frac{2 D}{v l}\right)^{1 / 2}
$$

There are some important dimensionless numbers in the chemical engineering field, such as Reynolds number, Prandtl number as well as Peclet number. Among these important here is Peclet number in view of equation (14).

$$
P_{z}=v l / D
$$

in which $P_{z}$ is axial Peclet number and subscript $z$ means that it is the process in the axial direction. From equations (13) and (14), the relationship is developed between relative dispersion and axial Peclet number;

$$
\frac{\Delta \pi_{2}^{1 / 2}}{\Delta l}=\left(\frac{2}{P_{z}^{1 / 2}}\right)^{1 / 2}
$$

Axial Peclet number is known as an indicator as to which is more contributing to the determination of mass transport in a flowing system, convective process or effective diffusion.

It was argued by Norwich and Zelin (1970) that in a relatively complicated circulatory system that has capillaries in it, effective diffusion can be achieved by two different processes; (i) virtual diffusion, and (ii) diffusion due to path-length difference.

$$
D_{\text {effective }}=D_{\text {virtual }}+D_{\text {path-length }}
$$

It was demonstrated by Bassingthwaighte et al. (1966) that relative dispersion is constant for a simple circulatory system, such as aorta or branching artery even with changing flow by 6 times. This means that Peclet number is also constant under such conditions. It can be considered that in a simple circulatory system, path-length is single, so that the difference in path-length is negligible. Therefore, diffusion effect is single, and there is $D_{\text {virtual }}$ only. Constant relative dispersion for aorta implies that $D_{\text {virtual }}$ is not altered by changing flow. By contrast, it is known (Coulam et al. 1966; Bassingthwaighte et al. 1966) that the more complicated systems have always a greater relative dispersion or a smaller Peclet number. Thus, relative dispersion of a circulatory system containing capillaries is made not only by virtual diffusion but also by difference in path-length.

From equations (11) and (13), we obtain equation (17).

$$
D_{\text {effective }} / l^{2}=(1 / 2)\left(\Delta \pi_{2} / \Delta t^{3}\right)
$$


From equations (16) and (17), we get equation (18).

$$
D_{\text {path-length }}=(1 / 2) l^{2}\left(\Delta \pi_{2} / \Delta \bar{t}^{3}\right)-D_{\text {virtual }}
$$

in which $D_{\text {virtual }}$ is given by;

$$
\left.\frac{D_{\text {virtual }}}{l^{2}}=\frac{1}{2} \text { (relative dispersion }\right)^{2} \frac{1}{\Delta t}
$$

where relative dispersion is constant, that is, about 0.2 after Bassingthwaighte (1966). From equations (18) and (19), we get equation (20).

$$
\frac{D_{\text {path-length }}}{l^{2}}=\frac{1}{2}\left[\frac{\Delta \pi_{2}}{\Delta \bar{t}^{3}}-0.2^{2} \frac{1}{\Delta \bar{t}}\right]
$$

Thus, the change in the path-length distribution may be evaluated per unit length averaged through the circulatory bed, from the sole measurement of the first two moments of $h(t)$.

The method to compute the moments of the transcoronary circulatory transport function and to estimate the changes in circulatory path-length distribution described in this paper is useful because of its simplicity in practice. Once coronary path-length distribution is known, it would be of great help for the clinical purpose in the assessment of the pathologic state in the heart of a patient and prediction of his prognosis. In this regard, one of the most interesting features would be the development of collateral circulations. Although they can be visualized by cine-coronary arteriography, limitation exists because the material is not physiologically inert. However, the significance of the momentanalysis of $h(t)$ is of limited value at present, because there is no way to measure the changes in the averaged vascular length. Under the conditions where length is considered to increase, however, any increase in the value calculated by the equation (20) indicates an increase in path-length distribution. For collateralized heart where retrograde flow appears with a large increase in the length in an average, and where the increase in the path-length distribution exceeds the former, then the equation (20) makes sense and becomes informative as to the pathologic process in the coronary circulation.

\section{Acknowledgment}

We would like to acknowledge the technical assistance of Mr. Eiji Tsuchida.

\section{References}

1) Bassingthwaighte, J.B. (1966) Plasma indicator dispersion in arteries of the human leg. Circulat. Res., 19, 332-346.

2) Bassingthwaighte, J.B. (1970) Blood flow and diffusion through mammalian organs. Science, 167, 1347-1353.

3) Bassingthwaighte, J.B. \& Ackerman, F.H. (1967) Mathematical linearity of circulatory transport. J. appl. Physiol., 22, 879-888.

4) Bassingthwaighte, J.B. Ackerman, F.H. \& Wood, E.H. (1966) Applications of the lagged normal density curve as a model for arterial dilution curves. Circulat. Res., $18,398-415$. 
5) Coulam, C.M., Warner, H.R., Wood, E.H. \& Bassingthwaighte, J.B. (1966) A transfer function analysis of coronary and renal circulation calculated from upstream and downstream indicator dilution curves. Circulat. Res., 19, 879-890.

6) Cramér, H. (1946) Mathematical Methods of Statistics. Princeton University Press, Princeton.

7) Gamel, J., Rousseau, W.F., Katholi, C.R. \& Mesel, E. (1973) Pitfalls in digital computation of the impulse response of vascular beds from indicator-dilution curves. Circulat. Res., 32, 516-523.

8) Kinsman, J.M., Moore, J.W. \& Hamilton, W.F. (1929) Studies on the circulation. 1. Injection method. Physical and mathematical considerations. Amer. J. Physiol., $89,322-330$.

9) Knopp, T.J. \& Bassingthwaighte, J.B. (1969) Effect of flow on transpulmonary circulatory transport functions. J. appl. Physiol., 27, 36-43.

10) Liedtke, A.J., Kemp, H.G., Borkenhagen, D.M. \& Gorlin R. (1973) Myocardial transit times from intracoronary dye dilution curves in normal subjects and patients with coronary artery disease. Amer. $J$. Cardiol, 32, 831-839.

11) Lilienfield, L.S. \& Kovach, R.D. (1956) Simplified method for calculating flow, mean circulation time and downslope from indicator-dilution curves. Proc. Soc. exp. Biol. Med. (N.Y.), 91, 595-598.

12) Meier, P. \& Zierler, K.L. (1954) On the theory of the indicator dilution method for the measurement of blood flow and volume. J. appl. Physiol., 6, 731-744.

13) Norwich, H.K. \& Zelin, S. (1970) The dispersion of indicator in the cardiopulmonary system. Bull. Math. Biophysics, 32, 25-43.

14) Stephenson, J.L. (1948) Theory of the measurement of blood flow by the dilution of an indicator. Bull. Math. Biophyscis, 10, 117-120.

15) Taylor, G. (1953) Dispersion of soluble matter in solvent flowing slowly through a tube. Proc. roy. Soc. A., 219, 186-203.

16) Wingard, L.B., Chorbajian, T. \& Galla, S.J. (1972) Concepts of residence time distribution applied to the indicator-dilution method. J. appl. Physiol, 33, 264-275. 